\title{
Mixed Halide Bulk Perovskite Triplet Sensitizers: Interplay between Band Alignment, Mid-gap Traps and Phonons
}

Alexander S. Bieber, ${ }^{1, \#}$ Zachary A. VanOrman, ${ }^{1, \#}$ Hayley K. Drozdick, ${ }^{1}$ Rachel Weiss, ${ }^{1}$ Sarah Wieghold, ${ }^{1,2,3}$ Lea Nienhaus ${ }^{1, *}$

${ }^{1}$ Department of Chemistry and Biochemistry, Florida State University, Tallahassee, FL 32306, USA

${ }^{2}$ Center for Nanoscale Materials, Argonne National Laboratory, Lemont, IL 60439, USA

${ }^{3}$ Advanced Photon Source, Argonne National Laboratory, Lemont, IL 60439, USA

\#equal contribution

*corresponding Author: lnienhaus@fsu.edu 


\begin{abstract}
Photon upconversion, particularly via triplet-triplet annihilation (TTA), could prove beneficial in expanding the efficiencies and overall impacts of optoelectronic devices across a multitude of technologies. The recent development of bulk metal halide perovskites as triplet sensitizers is one potential step toward the industrialization of upconversion-enabled devices. Here, we investigate the impact of varying additions of bromide into a lead iodide perovskite thin film on the TTA upconversion process in the annihilator molecule rubrene. We find an interplay between the bromide content and the overall device efficiency. In particular, a higher bromide content results in higher internal upconversion efficiencies, enabled by more efficient charge extraction at the interface, likely due to a more favorable band alignment. However, the external upconversion efficiency decreases, as the absorption cross section in the near infrared is reduced. The highest upconversion performance is found in our study for a bromide content of $5 \%$. This result can be traced back to a high absorption cross section in the near infrared and higher photoluminescence quantum yield in comparison to the iodide-only perovskite, as well as an increased driving force for charge transfer.
\end{abstract}




\section{INTRODUCTION}

The steady increase in the power conversion efficiency (PCE) of lead halide perovskite photovoltaics (PV), going from $3.8 \%$ to $25.5 \%$ in 12 years, has piqued significant interest, in large part due to their low cost and facile fabrication. ${ }^{1,2}$ Key factors in this rapid PCE increase are the unique properties of perovskites, including defect-tolerance, long charge carrier lifetime and diffusion lengths, high absorption cross sections and compositional bandgap and band energy tunability..$^{3,4}$

Due to the lower bandgap of iodide-based perovskites compared to other halide compositions, they are most commonly utilized for PV applications. However, the addition of small amounts of chloride or bromide into iodide-rich films has shown to increase the carrier lifetimes. The addition of bromide, in particular, has shown to be a viable route to fabricating pinhole-free and smooth perovskite thin films, improving the long-term stability, increasing carrier transport and diffusion lengths, and decreasing non-radiative recombination. ${ }^{5-7}$ While large amounts of bromide addition results in a significant blue-shift of the emission, and increase of the bandgap, modest bromide addition of up to $15 \%$ only slightly shifts the absorption onset.

Recently, in addition to their function as the absorber layer in perovskite PV, bulk lead iodide perovskites consisting of various amounts of methylammonium (MA) and formamidinium (FA) as the A-site cation have shown great promise as triplet sensitizers for photon upconversion (UC) in rubrene through triplet-triplet annihilation (TTA). The same properties which make perovskite materials uniquely qualified as the active layer in a PV, enable triplet sensitization through a freecharge carrier process. ${ }^{8-15}$

Photon UC in general describes the process of increasing the emitted photon energy upon photon absorption. ${ }^{16-20}$ To comply with energy conservation laws, this process requires the combination 
of the energy of multiple absorbed photons to generate a single high energy excited state. ${ }^{21,22}$ In TTA, this process occurs through the combination of long-lived triplet states, which enable the storage of the energy prior to the TTA-UC process. ${ }^{18,22,23}$

In bulk perovskite-sensitized UC, the triplet state is accessed by an asynchronous hole transfer from the valence band (VB) of the perovskite to the highest occupied molecular orbital (HOMO) of rubrene, coupled to an electron transfer from the conduction band $(\mathrm{CB})$ of the perovskite to form the bound triplet exciton. ${ }^{8,10}$ In the current iodide-only perovskite UC systems, the band alignment is such that hole transfer is strongly energetically favored, while electron transfer is nearly isoenergetic. The exact energy alignment depends on the MA/FA ratio used, which tunes the doping of the film (n-type vs. p-type), ${ }^{24}$ as well as the absolute energy levels of the VB/CB. Generally, a lower MA content results in shallower VB and CB levels, which can potentially increase the energetic driving force for electron transfer. ${ }^{25}$ However, this is counteracted by a higher absorption of the FA-rich perovskites, which we have previously shown to exhibit higher UC photoluminescence (PL) intensities than their MA-rich counterparts. Other important factors to consider are the native PL lifetimes and resulting carrier diffusion lengths, ${ }^{14}$ Fermi levels ${ }^{24}$ and interfacial band bending, ${ }^{11,26}$ which can all influence the number of charges extracted at the perovskite/rubrene interface. We have previously shown that one of the current limitations of the perovskite/rubrene UC system is incomplete charge extraction, which can be attributed to the lack of driving force for electron transfer, a limited driving force for charge transport to the interface, localized trap states or charge transfer states mediating the charge transfer., ${ }^{9,11,15,26}$

In this contribution, we investigate the effect of bromide addition up to $15 \%$ on the TTA-UC process in perovskite/rubrene systems. The bilayer UC devices consist of a $\sim 100 \mathrm{~nm}$ thin

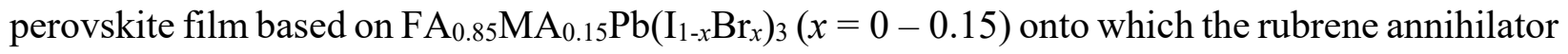


layer doped with $\sim 1 \%$ dibenzotetraphenylperiflanthene (DBP) is spin coated. ${ }^{27} \mathrm{We}$ find that small amounts of bromide increase the native perovskite PL quantum yield and lifetime, which result in an increased UC yield, due to the competition between interfacial carrier trapping and charge transfer to rubrene. ${ }^{26}$ Interestingly, we find that even sub-bandgap photons can be absorbed and subsequently upconverted, indicating the possible role of mid-gap trap states and thermal excitation to the band edge by the rich phonon bath in perovskites on the UC process. ${ }^{28-30}$

\section{RESULTS AND DISCUSSION}

The base perovskite composition used is $\mathrm{FA}_{0.85} \mathrm{MA}_{0.15} \mathrm{~Pb}\left(\mathrm{I}_{1-x}, \mathrm{Br}_{x}\right)_{3}$, where $x$ is varied from $0-0.15$. To elucidate the role of bromide addition on the TTA-UC process, bilayer UC devices are fabricated by spin coating a $10 \mathrm{mg} / \mathrm{mL}$ rubrene solution doped with $1 \%$ DBP onto the base perovskites. A post-fabrication thermal annealing step is utilized to increase the UC performance and drive off excess solvent. ${ }^{31}$
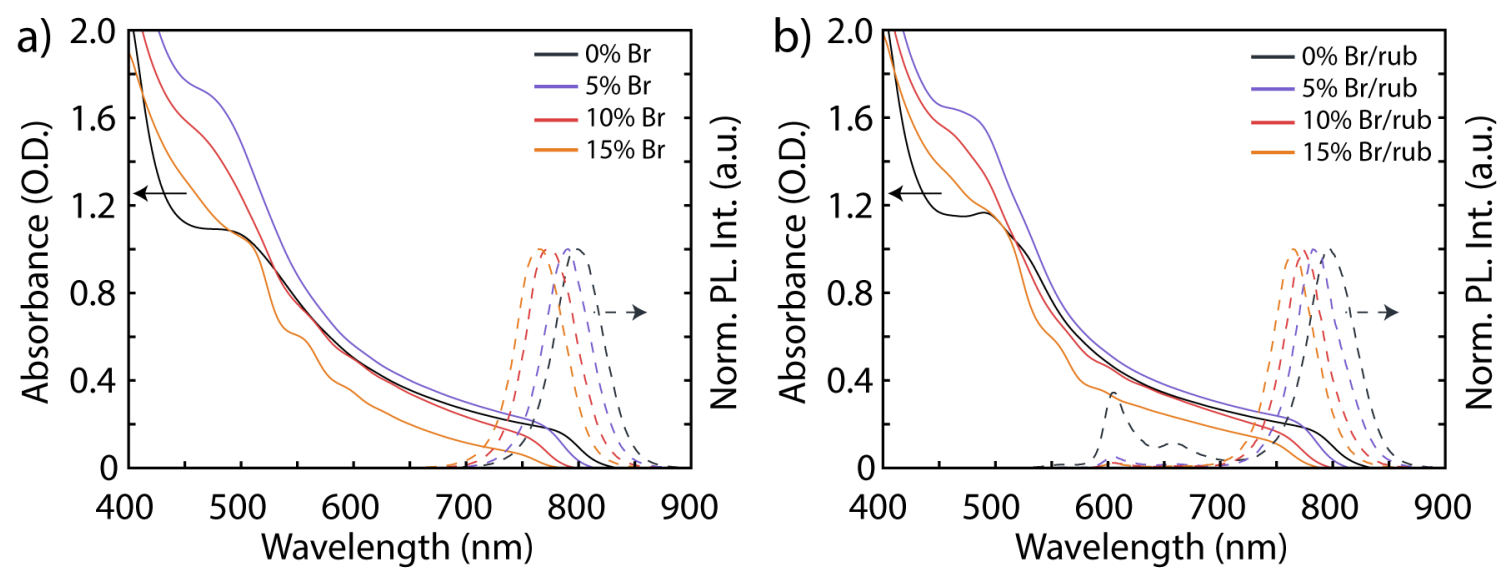

Figure 1: UV-vis absorbance (solid) and normalized PL (dashed) of the (a) perovskite-only films with varying bromide content and (b) the corresponding perovskite/rubrene bilayer films. The normalized PL was collected under $405 \mathrm{~nm}$ illumination. 
As expected, we find an increase in the bandgap upon $\mathrm{Br}$ addition, with a corresponding blue shift of the absorption onset and emission peak wavelength from $800 \mathrm{~nm}$ for the iodide-only perovskite $\mathrm{FA}_{0.85} \mathrm{MA}_{0.15} \mathrm{PbI}_{3}$ to $760 \mathrm{~nm}$ for $\mathrm{FA}_{0.85} \mathrm{MA}_{0.15} \mathrm{~Pb}\left(\mathrm{I}_{0.85}, \mathrm{Br}_{0.15}\right)_{3}$, as seen in Figure 1a. There are no additional shifts in the absorption onset or emission wavelength upon rubrene deposition, and both the rubrene/DBP and perovskite emission are clearly observed under $405 \mathrm{~nm}$ excitation (Figure $1 b)$.

A strong increase in the absolute PL intensity is seen upon bromide addition (Figure 2), highlighting the expected increase in radiative recombination caused by a reduced number of nonradiative carrier traps. Due to photodarkening of the perovskite under continuous illumination and halide segregation occurring (vide infra), the absolute PL intensity, however, must be taken with a grain of salt, as it just depicts a snapshot of the PL at a certain time after illumination. Halide segregation and the resulting red shift of the PL peak intensity is a known issue in mixed halide perovskite thin films. ${ }^{32,33}$ Halide migration under illumination results in phase-segregated bromide-rich and iodide-rich regions, the latter of which can act as charge carrier recombination centers, hindering the effective flow of charge carriers to the interface at which they are extracted. To infer whether halide migration has an influence on the UC intensity, we first investigate the effect of continuous illumination on the mixed halide perovskite compositions and corresponding UC devices presented here. Figure 2a,b depicts the perovskite emission under $405 \mathrm{~nm}$ excitation over the course of $120 \mathrm{~s}$ for the perovskite and the bilayer UC devices, respectively. 


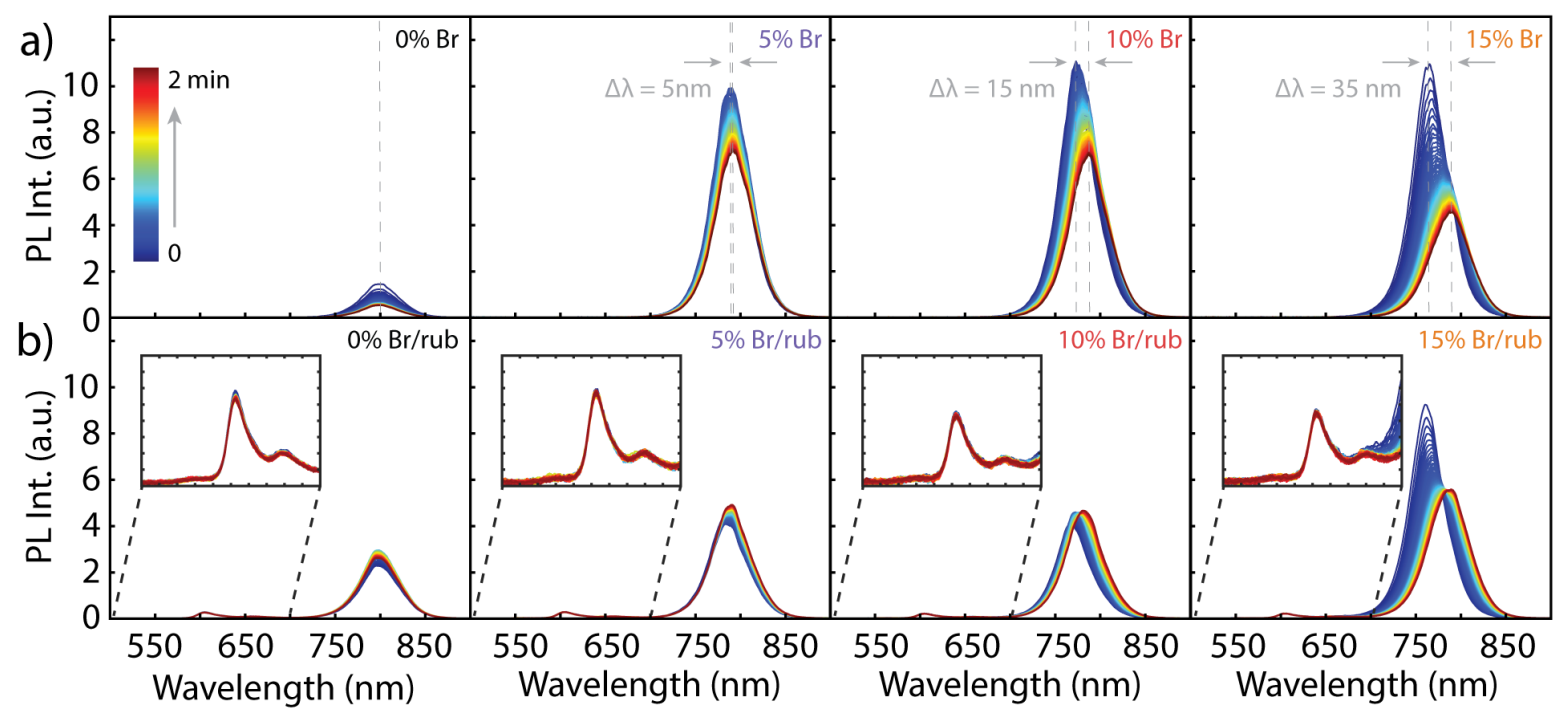

Figure 2: Time-dependent PL of (a) perovskite-only and (b) perovskite/rubrene bilayer films with varying bromide content. A zoom in of the rubrene emission is shown in the inset. The emission was collected under $405 \mathrm{~nm}$ excitation over a time period of 120 seconds. The initial peak position is shown by the leftmost dashed line, where at higher $\mathrm{Br}$ content, the rightmost dashed line yields the center peak position after halide migration. The difference between the two peak positions, in $\mathrm{nm}$, is given as $\Delta \lambda$.

We observe the characteristic red shift of the PL indicating halide segregation for the mixed halide perovskites. ${ }^{34-36}$ As expected, with increasing bromide concentration, the magnitude of the observed red shift increases: $\Delta \lambda=5 \mathrm{~nm}, \Delta \lambda=15 \mathrm{~nm}$ and $\Delta \lambda=35 \mathrm{~nm}$ for $5 \%, 10 \%$ and $15 \%$ bromide, respectively. A smaller red shift can be observed in the mixed halide perovskite PL intensity under $780 \mathrm{~nm}$ excitation (Figure S1), highlighting the expected effect of excitation photon energy on the halide migration process. Additionally, both photobrightening and photodarkening of the perovskite PL have been previously reported, ${ }^{37,38}$ as well as photodarkening followed by photobrightening. ${ }^{26}$ These effects are strongly dependent on the photon fluence, the excitation wavelength, as well as the local perovskite environment. ${ }^{37,38}$ Due to spot-to-spot variations in the relative PL behavior due to local inhomogeneity, ${ }^{26,39,40}$ the change in the absolute intensity of the perovskite PL is not considered here. No changes in the rubrene/DBP emission are found under direct excitation, indicating the emissive dye is stable and does not degrade under these conditions. 
Since the triplet sensitization is thought to occur through an asynchronous free charge carrier transfer from the perovskite to the triplet state of rubrene,${ }^{10}$ the band alignment is the key driving force for charge transfer to the triplet state of rubrene and the composition-dependent changes in the absolute $\mathrm{VB}$ and $\mathrm{CB}$ of the mixed halide perovskites are critical in this study. To first approximation, the addition of bromide is expected to shift the VB to a deeper level, and the CB to a shallower level, effectively increasing the driving force for both electron and hole transfer (Figure 3). ${ }^{25,32}$

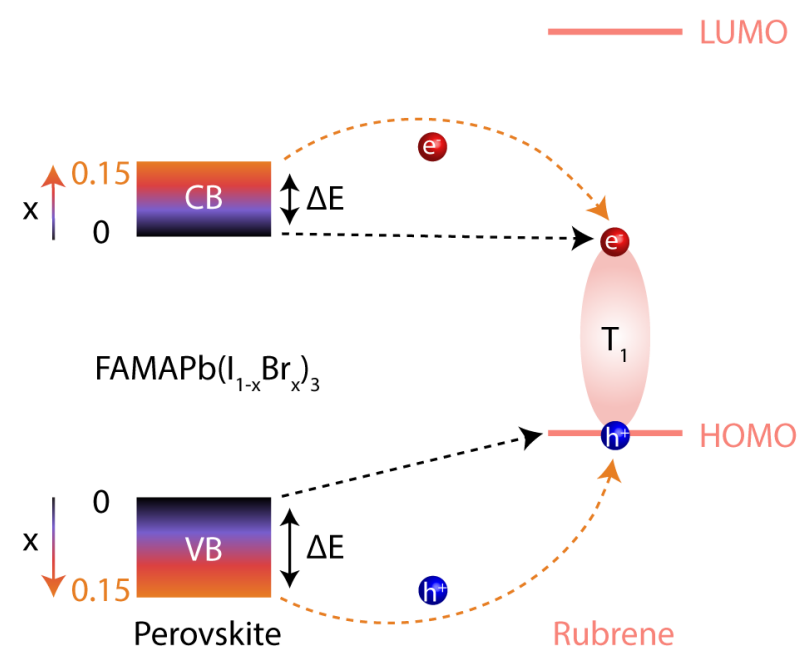

Figure 3: Schematic detailing the charge transfer process from the perovskite sensitizer to the rubrene annihilator, resulting in the population of the rubrene triplet state $\left(T_{1}\right)$. The addition of bromide (black to orange for increasing bromide content) changes the absolute band energies, resulting in a different energetic driving force for each composition for both hole and electron transfer.

A recent study also reports shallower energy levels for a related mixed halide perovskite: $\operatorname{MAPb}\left(\mathrm{I}_{0.875}, \mathrm{Br}_{0.125}\right){ }^{41}$ The $200 \mathrm{meV}$ shift of the $\mathrm{CB}, \mathrm{VB}$ and Fermi level observed in this system would also result in an increase in the driving force for electron transfer to the rubrene triplet, however, at the expense of a reduction of the driving force for hole transfer, which would still remain energetically favored. The predicted increase in driving force for charge transfer to the 
triplet state upon bromide addition, however, comes at the cost of a wider bandgap and thus, a reduced absorption in the near infrared spectral region. ${ }^{33,41}$

To gain deeper insight into the effect of the band alignment on the triplet sensitization, and quenching of the perovskite excited state, we turn to time-resolved PL spectroscopy. A slight decrease in the lifetime can be observed upon addition of 5\% bromide, however, with a corresponding increase in the amount of radiative decay, as observed in the increased absolute PL intensity (compare Figure 2). With further increasing bromide content, the average PL lifetimes become longer (Figure 4a and Table 1). In agreement with previous results, we find the amplitude of the rapid early time component to increase with increasing bromide concentration, while the lifetime at late time increases up to our highest bromide content of $15 \%$ (inset Figure $4 \mathrm{a}$ ). ${ }^{32}$ Due to the multiexponential behavior of the perovskite PL decay, we have fit each intensity decay to a triexponential as shown in Equation 1:

$$
I(t)=A_{1} \exp \left(-\frac{t}{\tau_{1}}\right)+A_{2} \exp \left(-\frac{t}{\tau_{2}}\right)+A_{3} \exp \left(-\frac{t}{\tau_{3}}\right)
$$

Table 1: Amplitudes (A) and decay time components $(\tau)$ and amplitude weighted average lifetime ( $\left.\tau_{\text {ave }}\right)$ for the perovskite-only PL decay for each bromide content.

\begin{tabular}{|c|c|c|c|c|c|c|c|}
\hline$x$ & $A_{1}$ & $\tau_{1}(\mathrm{~ns})$ & $A_{2}$ & $\tau_{2}(\mathrm{~ns})$ & $A_{3}$ & $\tau_{3}(\mathrm{~ns})$ & $\boldsymbol{\tau}_{\text {ave }}(\mathrm{ns})$ \\
\hline $0 \%$ & 0.21 & 25.6 & 0.54 & 170 & 0.15 & 365 & $\mathbf{1 7 0}$ \\
\hline $5 \%$ & 0.11 & 17.9 & 0.85 & 154 & 0.33 & 421 & $\mathbf{1 4 8}$ \\
\hline $10 \%$ & 0.27 & 5.3 & 0.24 & 214 & 0.42 & 468 & $\mathbf{2 6 7}$ \\
\hline $15 \%$ & 0.36 & 20.8 & 0.00016 & 100 & 0.42 & 889 & $\mathbf{4 8 8}$ \\
\hline
\end{tabular}

As all perovskite PL lifetimes are taken under $635 \mathrm{~nm}$ illumination and at a constant fluence of 2.8 $\mathrm{W} / \mathrm{cm}^{2}$, and thus, result in different carrier concentrations in the perovskite thin films due to different absorption cross sections based on the bromide content. These changes in the native 
lifetime are expected due to the carrier density-dependent recombination dynamics observed in perovskite thin films. ${ }^{42-44}$ With increasing carrier density, the early-time component becomes slower, due to reduced rapid non-radiative decay, while the late time dynamics show an increased rate of recombination due to a higher probability of recombination.
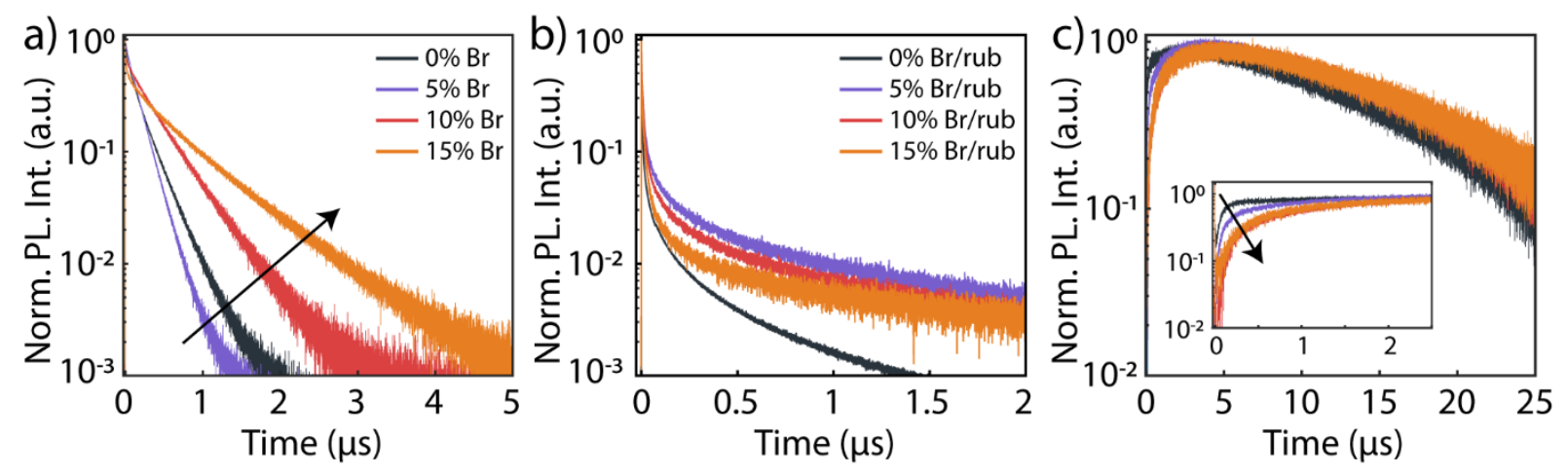

Figure 4: Time-resolved PL measurements. Perovskite PL decay dynamics of (a) perovskite-only films and (b) perovskite/rubrene bilayer films with different Br content. (c) UC PL decay dynamics of the perovskite/rubrene bilayer films. To monitor the PL decay dynamics of the perovskite, $635 \mathrm{~nm}$ excitation $\left(2.8 \mathrm{~W} / \mathrm{cm}^{2}\right)$, and the perovskite PL was monitored for $\lambda>700 \mathrm{~nm}$. The UC PL dynamics were measured under $780 \mathrm{~nm}$ excitation $\left(17 \mathrm{~mW} / \mathrm{cm}^{2}\right)$ power and monitored at $600 / 40 \mathrm{~nm}$ (center/width).

For all $\mathrm{FA}_{0.85} \mathrm{MA}_{0.15} \mathrm{~Pb}\left(\mathrm{I}_{1-x}, \mathrm{Br}_{x}\right)_{3} /$ rubrene bilayer devices, consistent with our previous results, we find quenching of the perovskite PL dynamics under $635 \mathrm{~nm}$ excitation (Figure 4b). ${ }^{10,26,45} \mathrm{We}$ observe similar early time dynamics for all compositions; however, the amplitude of this component shows spot-to-spot variations (Figure S2). The decay rate of the late time component is clearly dependent on the underlying composition. However, care must be taken when analyzing these results. For the related MA-rich perovskite/rubrene UC devices, we have shown that there is no direct correlation between the extent of early-time perovskite PL quenching and the UC PL intensity. Rather, we found that the less observable early time quenching, or the longer the average PL lifetime, the higher the UC PL intensity. ${ }^{26}$ The early time component is directly dependent on the underlying trap density, and carrier trapping and triplet sensitization have been shown to be competing pathways. ${ }^{14,26}$ 
Furthermore, perovskites exhibit power-dependent lifetimes: at lower carrier densities the early time component becomes faster, while the long-lived tail of the perovskite emission becomes longer. ${ }^{10,15}$ This general behavior is in agreement with our perovskite emission decays: once rubrene is added, the early time is quenched, while the lifetime at long times is elongated due to a lower carrier density, indicating efficient charge extraction.

In addition, the physical process of charge transfer to the rubrene triplet state is expected to be an ultrafast process on the sub-nanosecond timescale ${ }^{46,47}$ which cannot be captured within the time frame accessible by the time-correlated single-photon counting used here. Therefore, the detected early time component is more likely a convolution of 'slow' carrier migration to the interface and subsequent carrier extraction on the ultrafast timescale, as well as residual perovskite recombination well within the bulk of the material.

Based on these results, we expect that all perovskite compositions are capable of sensitizing the rubrene triplet state, due to the observed quenching of the PL lifetime. Interestingly, despite similar early time dynamics, we find the late time dynamics to become elongated with increasing bromide content, indicating a lower remaining carrier density. Considering the longer native lifetime of the perovskite with increasing bromide content, similar residual decay dynamics in the bilayer devices still correspond to large increases in the charge transfer efficiency to the rubrene triplet state. This increase in the number of charges transferred can be attributed to the increased carrier lifetimes in the mixed halide perovskite, enabling a longer carrier diffusion length prior to recombination or charge trapping, as well as the increased driving force for charge transfer at the interface due to the more favorable band alignment.

Lastly, to ensure the dynamics of the rubrene layer are not affected by the addition of bromide, we report the dynamics of the upconverted emission (Figure 4c). Here, a characteristic rise and fall in 
the PL dynamics is observed, as the emissive singlet population is rate-limited by the diffusionmediated TTA process. We have previously reported population-dependent rise dynamics, a result of two varying types of TTA: rapid TTA near the interface, and diffusion-mediated TTA far from the interface. ${ }^{45}$

Interestingly, the brightest $\mathrm{UC}$ is found for $\mathrm{FA}_{0.85} \mathrm{MA}_{0.15} \mathrm{~Pb}\left(\mathrm{I}_{0.85}, \mathrm{Br}_{0.15}\right)_{3}$, which exhibits less early time rise than $\mathrm{FA}_{0.85} \mathrm{MA}_{0.15} \mathrm{PbI}_{3}$. This suggests that the different interfacial band alignment upon bromide addition allows more carriers/triplets to diffuse, indicating that charges are not as localized to the interface as in the iodide-only composition. We further find a correlation between the fraction of the rapid rise component and the bromide content: less rapid rise component is found for higher bromide concentrations. This observation can simply be traced back to the varying amounts of absorption at the excitation wavelength at $780 \mathrm{~nm}$ (compare Supplementary Material Table S1): lower absorption results in less triplet states, resulting in more diffusion mediated TTA further from the interface.

To further investigate the UC properties of our bilayer devices, we determine the steady-state UC PL intensity under $780 \mathrm{~nm}$ excitation. The visible PL intensity under $780 \mathrm{~nm}$ excitation is related to the external UC efficiency, defined as the number of incident photons converted to UC photons. The external UC quantum yield (QY) is dependent on the efficiency of triplet sensitization $\phi_{T E T}$, the efficiency of TTA $\phi_{T T A}$ and the QY of the annihilator $\phi_{a n n}$. In contrast to previous excitonic systems, ${ }^{18,48}$ the efficiency of intersystem crossing $\phi_{I S C}$ can be ignored as this process does not occur in the perovskite-based TTA-UC system presented here:

$$
\phi_{U C}=\phi_{T E T} \phi_{T T A} \phi_{a n n} .
$$

Both $\phi_{T T A}$ and $\phi_{a n n}$ are specific to the annihilator, which is the same in all cases, hence the differences in observed $\phi_{U C}$ can only be related to $\phi_{T E T}$. As we have previously shown, the 
absolute external UC quantum efficiency $\left(\phi_{U C}\right)$ is difficult to obtain for perovskite-based UC systems due to effects of perovskite photobrightening resulting from trap filling, particularly at low solar-relevant fluences. ${ }^{26}$ As a result, we refrain from reporting absolute values and just focus on relative PL intensities.

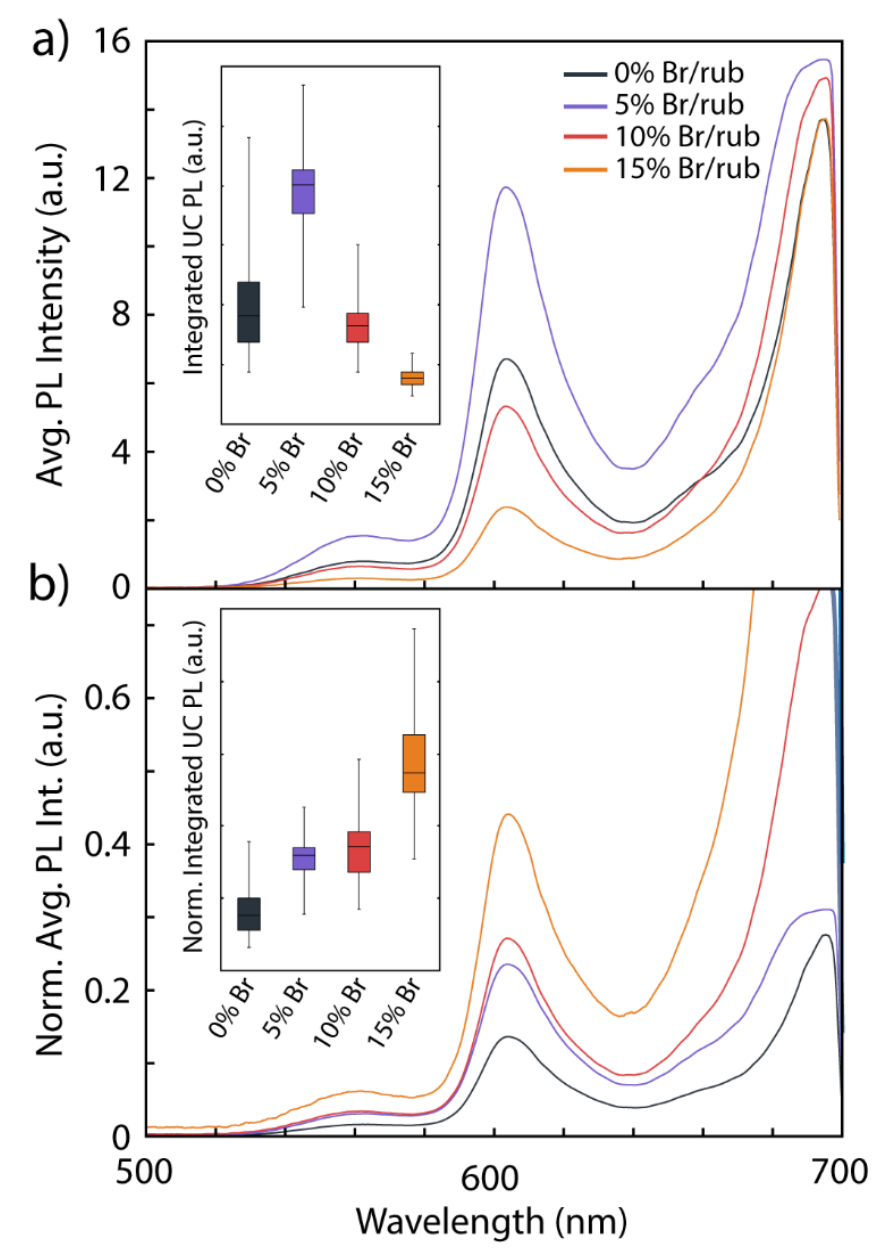

Figure 5: (a) Average UC PL intensity of the perovskite/rubrene films with varying bromide content. b) Normalized average UC PL intensity of the perovskite/rubrene films by the overlap integral of the film absorbance and the 780 $\mathrm{nm}$ laser line. In both (a) and (b), the spectra shown are the average of 20 spectra taken at various spots on two independent sample sets. The inset shows the box plots of the integrated UC PL intensity for each spectrum.

Under $780 \mathrm{~nm}$ excitation, to first approximation, we would expect only the compositions which have a bandgap larger than $1.59 \mathrm{eV}(780 \mathrm{~nm})$ to be capable of UC. However, we find that all compositions show strong UC PL, as shown in Figure 5a and Figure S3. For additional statistics, 
two independent sample sets are compared. The inset in Figure 5a shows a box plot of the integrated UC PL intensity (integrated from $500-630 \mathrm{~nm}$ ) taken at 20 spots across each sample, highlighting the heterogeneity of the fabricated samples. We find the highest UC PL intensity for $\mathrm{FA}_{0.85} \mathrm{MA}_{0.15} \mathrm{~Pb}\left(\mathrm{I}_{0.95}, \mathrm{Br}_{0.05}\right)_{3}$, which can be attributed to a combination of the increased driving force for electron extraction and elongated charge carrier recombination dynamics, with a strong underlying absorption at $780 \mathrm{~nm}$. The second highest UC PL intensity is found for the iodide only $\mathrm{FA}_{0.85} \mathrm{MA}_{0.15} \mathrm{PbI}_{3}$. Beyond the $5 \%$ bromide content in $\mathrm{FA}_{0.85} \mathrm{MA}_{0.15} \mathrm{~Pb}\left(\mathrm{I}_{0.95}, \mathrm{Br}_{0.05}\right)_{3}$, we find an increasing bromide concentration to yield a lower $\phi_{U C}$, respectively.

To investigate the possible effect of halide migration on the UC PL, we track the UC PL intensity under continuous illumination for $120 \mathrm{~s}$ (Figure S1b). We do not observe a change in the UC PL intensity for the low bromide content of up to $15 \%$ based on halide migration. Rather, the previously observed dependence of the UC PL intensity on the underlying perovskite PL intensity is found: if the underlying perovskite photobrightens, the UC PL increases, while a photodarkening of the underlying perovskite PL results in a decrease of the UC PL intensity. This indicates that the slight effects of halide segregation under $780 \mathrm{~nm}$ excitation in the perovskite thin films including up to $15 \%$ bromide do not influence the charge transfer properties to the rubrene triplet level on the ensemble level investigated here. Rather it is even possible that halide segregation can 'concentrate' the charge carriers in iodide-rich domains, which are located at the interface, resulting in an increase in probability of transfer to the triplet state of rubrene.

However, to clearly understand the effect of the UC PL intensity on the underlying bromide content, in particular, to understand the effect of the band alignment on $\phi_{T E T}$, the changes in the absorption at the excitation wavelength must be considered. To first approximation, based on our hypothesis that the band alignment is the critical factor in the charge extraction process, we would 
expect the highest bromide content to yield the highest UC efficiency, relative to the number of absorbed photons. Normalizing the UC PL intensity by the overlap integral of the perovskite absorption and the excitation laser line shape $J_{\text {overlap }}$ (compare Table S1) yields the UC PL intensity based relative to the absorbance of the perovskite thin film, a metric related to the internal UC quantum efficiency $\left(\phi_{U C_{-} I n t}\right)$ :

$$
\phi_{U C_{-} I n t}=\frac{\phi_{T E T} \phi_{T T A} \phi_{a n n}}{J_{\text {overlap }}} .
$$

Indeed, we find the perovskite composition with the highest bromide content $\mathrm{FA}_{0.85} \mathrm{MA}_{0.15} \mathrm{~Pb}\left(\mathrm{I}_{0.85}, \mathrm{Br}_{0.15}\right)_{3}$ to exhibit the highest $\phi_{U C_{-} \text {Int }}$ (Figure $5 \mathrm{~b}$ ). This confirms our hypothesis that the underlying perovskite PL recombination dynamics and energetic driving force for concurrent electron and hole extraction are the key factors limiting the UC efficiency in perovskite/rubrene UC devices.

Lastly, we address the question how it is possible to excite the mixed halide perovskites using 780 $\mathrm{nm}$ excitation, which is slightly sub-bandgap in energy for the higher bromide concentrations of $10 \%$ and $15 \%$, which emit at $775 \mathrm{~nm}$ and $760 \mathrm{~nm}$, respectively. This observation is directly related to the defect tolerance of perovskite materials, and the rich defect manifold available within the bandgap. Traps in perovskites are classified as deep or shallow traps, with the distinction that carriers caught in shallow traps can be de-trapped by the ambient thermal energy $k T$. In addition to trap states caused by atomic defects, Urbach tails stemming from lattice disorder, doping or thermal motion can result in shallow traps found within the bandgap ${ }^{49-52}$ Direct pumping of shallow traps has previously been reported in perovskites, ${ }^{49}$ and the rich phonon bath present in the bulk perovskite enables rapid thermal excitation from the sub-bandgap trap states at $\sim 1.59 \mathrm{eV}$ to the band edge at $1.63 \mathrm{eV}$ for $\mathrm{FA}_{0.85} \mathrm{MA}_{0.15} \mathrm{~Pb}\left(\mathrm{I}_{0.85}, \mathrm{Br}_{0.15}\right)_{3} .^{28,29}$ The observation of the band edge $\mathrm{FA}_{0.85} \mathrm{MA}_{0.15} \mathrm{~Pb}\left(\mathrm{I}_{0.85}, \mathrm{Br}_{0.15}\right)_{3} \mathrm{PL}$ under $780 \mathrm{~nm}$ pump is a clear indication that this thermal 
excitation is occurring (Figure 5). To support our hypothesis that the band edge levels, rather than sub-bandgap traps, are the mediating factors for charge transfer to the rubrene triplet state, we refer back to the $\mathrm{PL}$ dynamics of $\mathrm{FA}_{0.85} \mathrm{MA}_{0.15} \mathrm{~Pb}\left(\mathrm{I}_{0.85}, \mathrm{Br}_{0.15}\right)_{3}$ in presence of rubrene (vide supra). If charges were not thermally excited to the band edge, we would not be able to track them via PL. In addition, if the charges were extracted to the triplet state of rubrene prior to their thermal excitation to the emissive band edge, we would not expect to see strong effects in the PL decay dynamics. Once the band edge is populated via thermal excitation, subsequent charge extraction enables the population of the triplet state of rubrene, which results in the observed TTA-UC process. A summary of the ongoing processes is shown in Figure 6.

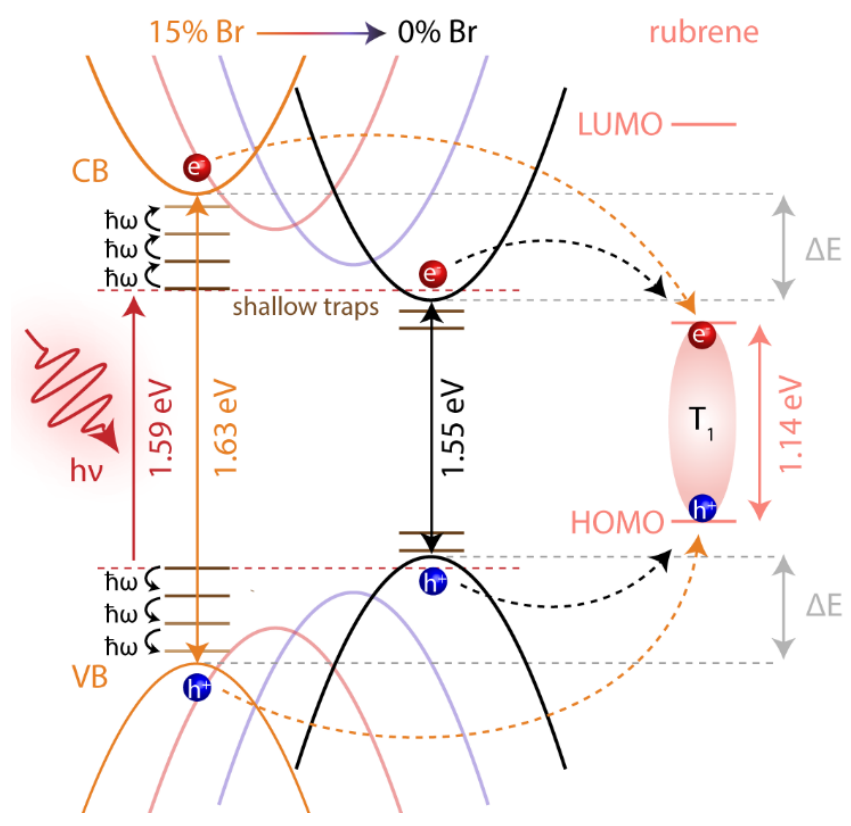

Figure 6: Schematic detailing the charge transfer process from the perovskite to the rubrene triplet state for various bromide contents. For higher bromide contents (15\%), optical excitation results in the population of sub-bandgap shallow trap states. Phonon-mediated thermal excitation enables the population of the band edge before charge extraction to rubrene.

\section{CONCLUSIONS}

In summary, the effect of the halide composition on the TTA-UC process in rubrene has been investigated by changing the bromide fraction from $x=0-0.15$. The increased carrier 
recombination lifetimes observed in mixed halide perovskite thin films with increasing bromide content, combined with a more beneficial band alignment enable a more efficient charge extraction to the triplet state of rubrene, as observed in the higher internal UC efficiency $\phi_{U C_{-} I n t}$.

From a practical device perspective however, the external UC efficiency $\phi_{U C}$ is the relevant metric. Only absorbed photons can be upconverted through the TTA process. We find that slight blue shifts of the perovskite bandgap due to $5 \%$ bromide content are counteracted by the increased driving force for charge transfer to the rubrene triplet state, thus enable a higher $\phi_{U C}$ than in the iodide-only counterpart. Despite more efficient charge extraction and a higher internal $\phi_{U C_{-} I n t}$, higher bromide contents substantially reduce the absorption in the near-infrared spectral region, thus, limit the achievable apparent anti-Stokes shift in the TTA-UC process.

Halide segregation does not have a detectable influence on the UC properties investigated on the ensemble level here. However, further studies with a higher spatial resolution approaching the diffraction limit may yield more localized information on the role of phase segregation on the PL properties on the sub-micron scale.

More generally, the results presented here indicate that the the main limiting factors in the efficiency of perovskite-sensitized TTA-UC are the energetic alignment between the perovskite sensitizer and the triplet acceptor and the defect density of the bulk perovskite thin film. To date, only the annihilator rubrene has successfully been utilized in perovskite-sensitized TTA-UC. This is in large part due to restrictions in annihilators capable of efficient solid-state UC, as well as a lack of other known annihilators with a proper band alignment with the perovskite sensitizers. Future efforts should be focused on finding new sensitizer/annihilator pairs with a more beneficial band alignment, while minimizing the energetic losses for interfacial charge extraction. 


\section{EXPERIMENTAL METHODS}

Device fabrication. Borosilicate glass substrates (University Wafer) were cleaned by sonication in solvents and UV ozone treatement (Ossila) prior to perovskite film deposition. All precursor solutions were prepared from a 1.2 $\mathrm{M} \mathrm{PbI}_{2}$ (TCI) and 1.2 $\mathrm{M} \mathrm{PbBr}_{2}$ stock solution in anhydrous DMF:DMSO (9:1, v:v, Sigma Aldrich). 1.2 M MAI (Dyenamo), 1.2 M FAI (Dyenamo) and 1.2 M MABr (Dyenamo) were then mixed with the respective stock solution in a 1:1.09 ratio. To obtain the various mixed-halide compositions, different ratios of these solutions were used. The films were spin-coated at $1000 \mathrm{rpm}$ for $10 \mathrm{~s}$ and $5000 \mathrm{rpm}$ for $30 \mathrm{~s}$. During the second spin-coating stage, chlorobenzene (Sigma Aldrich) was used as an antisolvent. The films were subsequently annealed at $110^{\circ} \mathrm{C}$ for $45 \mathrm{~min}$.

Rubrene (99.99\%) and DBP (98\%) were purchased from Sigma Aldrich and used as received without further purification. A solution of $10 \mathrm{mg} / \mathrm{mL}$ rubrene was prepared in anhydrous toluene (Sigma Aldrich) and doped with DBP at a concentration of $\sim 1 \%$. The rubrene/DBP solution was deposited by spin-coating onto the perovskite thin film at $6000 \mathrm{rpm}$ for 20 seconds. The resulting bilayers were annealed at $100{ }^{\circ} \mathrm{C}$ for 10 min. The upconversion devices were sealed with a coverslip using a 2-part epoxy (Devcon) under nitrogen atmosphere $\left(<0.5 \mathrm{ppm} \mathrm{O}_{2}\right)$ prior to removal from the glovebox.

\section{Optical characterization.}

UV-Vis absorption spectra were collected using a Thermo Scientific Evolution 220 spectrophotometer. The steady-state and time-dependent emission spectra were collected using $405 \mathrm{~nm}$ (LDH-D-C-405, PicoQuant) continuous wave excitation at a power density of $92 \mathrm{~W} / \mathrm{cm}^{2}$ with a $425 \mathrm{~nm}$ long pass filter (Chroma Tech) to remove excess laser scatter. The upconverted emission and time-dependent emission spectra were collected under continuous wave excitation 
using a $780 \mathrm{~nm}$ laser (LDH-D-C-780, PicoQuant) at a power density of $143 \mathrm{~W} / \mathrm{cm}^{2}$. Excess laser scatter was removed with a $700 \mathrm{~nm}$ short pass filter (ThorLabs) or a $780 \mathrm{~nm}$ notch filter (ThorLabs). An Ocean Insight emission spectrometer (HR2000+ES) was used to record the emission. Time-resolved PL decays were measured using a $635 \mathrm{~nm}$ pulsed laser (LDH-P-C$635 \mathrm{M}$, PicoQuant) at a repetition frequency of $125 \mathrm{kHz}$ and an average power density of 2.8 W/ $/ \mathrm{cm}^{2}$. A $633 \mathrm{~nm}$ notch filter (ThorLabs) was used to remove excess laser scatter and a $700 \mathrm{~nm}$ long pass filter (ThorLabs) was used to isolate the perovskite emission. UC PL dynamics were measured with a pulsed $780 \mathrm{~nm}$ laser (LDH-D-C-780, PicoQuant) at a repetition frequency of $31.25 \mathrm{kHz}$ and an average power density of $17 \mathrm{~mW} / \mathrm{cm}^{2}$. Photon arrival times were recorded using a MultiHarp 150 event timer (PicoQuant) connected to a silicon single-photon avalanche photodiode (Micro Photon Devices). The incident laser beam power was measured using a silicon power meter (ThorLabs PM100-D). Spot sizes were measured using the razor blade method $(90: 10)$.

\section{SUPPLEMENTARY MATERIAL}

See the supplementary material for additional figures and data as detailed in the text.

\section{ACKNOWLEDGEMENT}

The authors acknowledge funding by Florida State University. Use of the Center for Nanoscale Materials, an Office of Science user facility, was supported by the U.S. Department of Energy, Office of Science, Office of Basic Energy Sciences, under Contract No. DE-AC02-06CH11357. 


\section{CONFLICT OF INTEREST}

FSU has filed a provisional application for a US patent based on this technology that names L.N. and S.W. as inventors (Patent App. 16/983147).

\section{DATA AVAILABILITY}

The data that support the findings of this study are available from the corresponding author upon reasonable request.

\section{REFERENCES}

${ }^{1}$ M.A. Green, A. Ho-Baillie, and H.J. Snaith, Nat. Photonics 8, 506 (2014).

2 J.Y. Kim, J.-W. Lee, H.S. Jung, H. Shin, and N.-G. Park, Chem. Rev. 120, 7867 (2020).

${ }^{3}$ J.-P. Correa-Baena, M. Saliba, T. Buonassisi, M. Grätzel, A. Abate, W. Tress, and A. Hagfeldt, Science 358, 739 (2017).

${ }^{4}$ J.-P. Correa-Baena, Y. Luo, T.M. Brenner, J. Snaider, S. Sun, X. Li, M.A. Jensen, N.T.P. Hartono, L. Nienhaus, S. Wieghold, J.R. Poindexter, S. Wang, Y.S. Meng, T. Wang, B. Lai, M.V. Holt, Z. Cai, M.G. Bawendi, L. Huang, T. Buonassisi, and D.P. Fenning, Science 363, 627 (2019).

${ }^{5}$ N.J. Jeon, J.H. Noh, W.S. Yang, Y.C. Kim, S. Ryu, J. Seo, and S.I. Seok, Nature 517, 476 (2015).

${ }^{6}$ T.J. Jacobsson, J.-P. Correa-Baena, M. Pazoki, M. Saliba, K. Schenk, M. Grätzel, and A. Hagfeldt, Energy Environ. Sci. 9, 1706 (2016).

${ }^{7}$ E.M. Talbert, H.F. Zarick, N.J. Orfield, W. Li, W.R. Erwin, Z.R. DeBra, K.R. Reid, C.P. McDonald, J.R. McBride, J. Valentine, S.J. Rosenthal, and R. Bardhan, RSC Adv. 6, 86947 (2016).

${ }^{8}$ L. Nienhaus, J.-P. Correa-Baena, S. Wieghold, M. Einzinger, T.-A. Lin, K.E. Shulenberger, N.D. Klein, M. Wu, V. Bulović, T. Buonassisi, M.A. Baldo, and M.G. Bawendi, ACS Energy Lett. 888 (2019).

${ }^{9}$ Z.A. VanOrman and L. Nienhaus, ACS Energy Lett. 6, 3686 (2021).

${ }^{10}$ S. Wieghold, A.S. Bieber, Z.A. VanOrman, L. Daley, M. Leger, J.-P. Correa-Baena, and L. Nienhaus, Matter 1, 705 (2019).

${ }^{11}$ S. Wieghold and L. Nienhaus, J. Phys. Chem. Lett. 11, 601 (2020).

${ }^{12}$ Z.A. VanOrman, H.K. Drozdick, S. Wieghold, and L. Nienhaus, J. Mater. Chem. C 9, 2685 (2021).

${ }^{13}$ S. Wieghold, Z.A. VanOrman, and L. Nienhaus, Adv. Opt. Mater. 9, 2001470 (2021). 
${ }^{14}$ K. Prashanthan, B. Naydenov, K. Lips, E. Unger, and R.W. MacQueen, J. Chem. Phys. 153, $164711(2020)$.

${ }^{15}$ L. Wang, J.J. Yoo, T.-A. Lin, C.F. Perkinson, Y. Lu, M.A. Baldo, and M.G. Bawendi, Adv. Mater. 33, 2100854 (2021).

${ }^{16}$ F. Auzel, Chem. Rev. 104, 139 (2004).

${ }^{17}$ R.R. Islangulov, D.V. Kozlov, and F.N. Castellano, Chem. Commun. 3776 (2005).

18 T.N. Singh-Rachford and F.N. Castellano, Coord Chem Rev 254, 2560 (2010).

19 J. Zhou, Q. Liu, W. Feng, Y. Sun, and F. Li, Chem. Rev. 115, 395 (2015).

${ }^{20}$ C. Ye, L. Zhou, X. Wang, and Z. Liang, Phys. Chem. Chem. Phys. 18, 10818 (2016).

${ }^{21}$ Y.Y. Cheng, T. Khoury, R.G.C.R. Clady, M.J.Y. Tayebjee, N.J. Ekins-Daukes, M.J. Crossley, and T.W. Schmidt, Phys. Chem. Chem. Phys. 12, 66 (2010).

${ }^{22}$ T.W. Schmidt and F.N. Castellano, J. Phys. Chem. Lett. 5, 4062 (2014).

${ }^{23}$ Y.Y. Cheng, B. Fückel, T. Khoury, R.G.C.R. Clady, M.J.Y. Tayebjee, N.J. Ekins-Daukes, M.J. Crossley, and T.W. Schmidt, J. Phys. Chem. Lett. 1, 1795 (2010).

${ }^{24}$ C.C. Stoumpos, C.D. Malliakas, and M.G. Kanatzidis, Inorg Chem 52, 9019 (2013).

${ }^{25}$ S. Tao, I. Schmidt, G. Brocks, J. Jiang, I. Tranca, K. Meerholz, and S. Olthof, Nat. Commun. 10, 2560 (2019).

${ }^{26}$ Z.A. VanOrman, J. Lackner, S. Wieghold, K. Nienhaus, G.U. Nienhaus, and L. Nienhaus, Appl. Phys. Lett. 118, 203903 (2021).

${ }^{27}$ S. Wieghold, A.S. Bieber, Z.A. VanOrman, A. Rodriguez, and L. Nienhaus, J. Phys. Chem. C (2020).

${ }^{28}$ D.M. Monahan, L. Guo, J. Lin, L. Dou, P. Yang, and G.R. Fleming, J. Phys. Chem. Lett. 8, 3211 (2017).

${ }^{29}$ Y. Yang, D.P. Ostrowski, R.M. France, K. Zhu, J. van de Lagemaat, J.M. Luther, and M.C. Beard, Nat. Photonics 10, 53 (2016).

${ }^{30}$ J. Fu, Q. Xu, G. Han, B. Wu, C.H.A. Huan, M.L. Leek, and T.C. Sum, Nat. Commun. 8, 1300 (2017).

${ }^{31}$ A.S. Bieber, Z.A. VanOrman, S. Wieghold, and L. Nienhaus, J. Chem. Phys. 153, 084703 (2020).

${ }^{32}$ B. Park, B. Philippe, S.M. Jain, X. Zhang, T. Edvinsson, H. Rensmo, B. Zietz, and G. Boschloo, J. Mater. Chem. A 3, 21760 (2015).

${ }^{33}$ E.T. Hoke, D.J. Slotcavage, E.R. Dohner, A.R. Bowring, H.I. Karunadasa, and M.D. McGehee, Chem. Sci. 6, 613 (2015).

${ }^{34}$ A.J. Barker, A. Sadhanala, F. Deschler, M. Gandini, S.P. Senanayak, P.M. Pearce, E. Mosconi, A.J. Pearson, Y. Wu, A.R. Srimath Kandada, T. Leijtens, F. De Angelis, S.E. Dutton, A.

Petrozza, and R.H. Friend, ACS Energy Lett. 2, 1416 (2017).

${ }^{35}$ P. Gratia, G. Grancini, J.-N. Audinot, X. Jeanbourquin, E. Mosconi, I. Zimmermann, D.

Dowsett, Y. Lee, M. Grätzel, F. De Angelis, K. Sivula, T. Wirtz, and M.K. Nazeeruddin, J. Am. Chem. Soc. 138, 15821 (2016).

${ }^{36}$ P.V. Kamat and M. Kuno, Acc. Chem. Res. 54, 520 (2021).

${ }^{37}$ Z. Andaji-Garmaroudi, M. Anaya, A.J. Pearson, and S.D. Stranks, Adv. Energy Mater. 10, 1903109 (2020).

${ }^{38}$ W.-A. Quitsch, D.W. deQuilettes, O. Pfingsten, A. Schmitz, S. Ognjanovic, S. Jariwala, S. Koch, M. Winterer, D.S. Ginger, and G. Bacher, J. Phys. Chem. Lett. 9, 2062 (2018).

${ }^{39}$ S. Wieghold, A.S. Bieber, J. Lackner, K. Nienhaus, G.U. Nienhaus, and L. Nienhaus, ChemPhotoChem 4, 704 (2020). 
${ }^{40}$ S. Wieghold, J.-P. Correa-Baena, L. Nienhaus, S. Sun, K.E. Shulenberger, Z. Liu, J.S. Tresback, S.S. Shin, M.G. Bawendi, and T. Buonassisi, ACS Appl. Energy Mater. 1, 6801 (2018).

${ }^{41}$ M. Daboczi, S.R. Ratnasingham, L. Mohan, C. Pu, I. Hamilton, Y.-C. Chin, M.A. McLachlan, and J.-S. Kim, ACS Energy Lett. 3970 (2021).

${ }^{42}$ S.D. Stranks, V.M. Burlakov, T. Leijtens, J.M. Ball, A. Goriely, and H.J. Snaith, Phys. Rev. Appl. 2, 034007 (2014).

${ }^{43}$ C. Wehrenfennig, M. Liu, H.J. Snaith, M.B. Johnston, and L.M. Herz, Energy Environ. Sci. 7, 2269 (2014).

44 J.S. Manser and P.V. Kamat, Nat. Photonics 8, 737 (2014).

${ }^{45}$ S. Wieghold, A.S. Bieber, Z.A. VanOrman, and L. Nienhaus, J. Phys. Chem. Lett. 3806 (2019).

${ }^{46}$ N. Droseros, B. Dänekamp, D. Tsokkou, P.P. Boix, and N. Banerji, APL Mater. 7, 041115 (2019).

${ }^{47}$ G. Xing, N. Mathews, S. Sun, S.S. Lim, Y.M. Lam, and M. Grätzel, Science 342, 344 (2013).

${ }^{48}$ Y. Zhou, F.N. Castellano, T.W. Schmidt, and K. Hanson, ACS Energy Lett. 5, 2322 (2020).

${ }^{49}$ V.M. Caselli, Z. Wei, M.M. Ackermans, E.M. Hutter, B. Ehrler, and T.J. Savenije, ACS Energy Lett. 5, 3821 (2020).

${ }^{50}$ C.M. Sutter-Fella, D.W. Miller, Q.P. Ngo, E.T. Roe, F.M. Toma, I.D. Sharp, M.C. Lonergan, and A. Javey, ACS Energy Lett. 2, 709 (2017).

${ }^{51}$ P. Qin, J. Zhang, G. Yang, X. Yu, and G. Li, J. Mater. Chem. A 7, 1824 (2019).

52 B. Galvani, D. Suchet, A. Delamarre, M. Bescond, F.V. Michelini, M. Lannoo, J.-F. Guillemoles, and N. Cavassilas, ACS Omega 4, 21487 (2019). 